\title{
FACTORS CONTRIBUTING TO WELL-BEING: COMPARING FUNCTIONAL SOMATIC SYMPTOM DISORDERS AND WELL-DEFINED AUTOIMMUNE DISORDERS
}

\author{
Kendra Hebert, \& Lisa Best \\ Department of Psychology, University of New Brunswick, Saint John, NB (Canada)
}

\begin{abstract}
Functional somatic symptom disorders (FSSDs) are defined by persistent and chronic bodily complaints without a pathological explanation. Mindfulness involves the focus on the present moment by noticing surroundings, thoughts, feelings, and events, being nonreactive, being non-judgemental, and self-accepting. Psychological flexibility (PF) involves a focus on the present and the prioritization of thoughts, emotions, and behaviours that align with individual values and goals (Francis et al., 2016). Although PF does not involve a mindfulness practice, the two constructs are related. Research indicates consistent reported positive associations between mindfulness, PF, psychological wellbeing, and medical symptoms. In this study, individuals with FSSDs (fibromyalgia, chronic fatigue syndrome) were compared to those with well-defined autoimmune illnesses (multiple sclerosis, rheumatoid arthritis; AD) to determine how psychosocial factors affect wellness. Participants $(\mathrm{N}=609)$ were recruited from social media and online support groups and completed questionnaires to assess physical health (Chang et al., 2006), psychological wellness (Diener et al., 1985), anxiety (Spitzer et al., 2006), depression (Martin et al., 2006), psychological flexibility, (Francis et al., 2016) and mindfulness (Droutman et al., 2018]. Results indicated that having an FSSD and higher depression was associated with both lower physical and psychological wellness. Interestingly, different aspects of psychological flexibility predicted physical and psychological wellness. These results suggest that different aspects of PF are associated with better physical and psychological health. As PF is modifiable, individuals with chronic conditions could receive training that could ultimately improve their overall health.
\end{abstract}

Keywords: Functional somatic symptom disorder, autoimmune disorder, well-being, mindfulness.

\section{Introduction}

For individuals facing incurable chronic illnesses, obtaining the highest possible quality of life (QoL) is essential and depends upon physical and psychological wellness as well as optimal social functioning (McCabe \& McKern, 2002). It is important to educate patients to help them learn and adopt effective coping strategies and improve access to physical and psychological support. Functional somatic symptom disorders (FSSD) are characterized by lasting bodily complaints such as pain and fatigue that do not have a pathological explanation (Henningsen et al., 2007). Although diagnosis is based on a process of symptom elimination (Henningsen et al., 2007), clear diagnostic criteria have been developed for the three most well-defined FSS illnesses: fibromyalgia syndrome (FMS), chronic fatigue syndrome (CFS), and irritable bowel syndrome (IBS; Henningsen et al., 2007). CFS is categorized by persistent or relapsing debilitating chronic fatigue and other non-specific symptoms (e. g., muscle weakness, pain, cognitive deficits) that have no other underlying cause. FMS is characterized by chronic widespread pain (Wolfe et al., 2010); fatigue, cognitive impairments, headaches, abdominal pain or cramps, and depression are non-specific symptoms (Wolfe et al., 2016). Given the wide variety of symptoms and lack of diagnostic tests, diagnosis of FSSDs is difficult and can result in patient frustration.

Although similar symptoms are associated with well-defined autoimmune diseases (AD), such as multiple sclerosis (MS) and rheumatoid arthritis (RA), clear medical tests support diagnosis. MS is an autoimmune disease characterized by chronic inflammation due to the immune system attacking myelin sheaths central nervous system axons (Compston \& Coles, 2002), which causes lesions that can affect all central nervous system function (Mohr \& Cox, 2001). Although symptoms vary greatly, vision problems, loss of function and feeling in limbs, bladder and bowel incontinence, pain, and loss of balance are common (Jellinger, 1999). Rheumatoid arthritis (RA) is a chronic inflammatory joint disease caused by 
infiltration of joints by $\mathrm{T}$ cells, $\mathrm{B}$ cells, and monocytes, destroying cartilage and bone leading to inflammation and damage (Aletaha \& Smolen, 2018). Symptoms include pain and swelling and misaligned joints (Sokka et al. 1999). Manifestations outside of joints can occur including rheumatoid nodules (firm subcutaneous lumps near bony prominences) and rheumatoid vasculitis (necrotizing inflammation of arteries; Aletaha \& Smolen, 2018). Individuals who have symptoms of MS or RA are typically referred for medical tests, with diagnosis occurring soon after test results are available.

\subsection{Modifiable factors that could improve physical and psychological wellness}

One of the main goals of CBT is to decrease pain and distress and increase both social and physical functioning (Turk et al., 1983). One CBT model is Acceptance and Commitment Therapy (ACT; Hayes et al., 2016). The goal of ACT is to increase psychological flexibility (PF; Hayes et al., 2016), which is a person's ability to detect and acknowledge interfering thoughts, emotions, and physical sensations without acting on them (Wicksell et al., 2012). PF facilitates appropriate behaviours that are aligned with personal values and long term-goals (Wicksell et al., 2012). After undergoing ACT, Wicksell and colleagues (2012) found improvements in self-efficacy, pain related functioning, depression, anxiety, and psychological inflexibility in FMS patients. Among FMS patients, a decrease in psychological inflexibility facilitated improvement in pain disability (Wicksell et al., 2012).

Mindfulness is a personal process that involves a focus on the present moment (Droutman et al., 2018) that is accomplished by noticing surroundings, thoughts, feelings and events, being nonreactive, being non-judgemental, and self-accepting (Droutman et al., 2018). It is both a regulated and maintained attention to existing sensory, emotional, and cognitive events that are changeable and transient, without allowing emotions to cloud judgement (Zeidan et al., 2012). Further, it can be developed by mental training, such as meditation, which changes how a person evaluates sensory events (Zeidan et al., 2012). The strong positive relationship between mindfulness and satisfaction with life has been well-established (Kong et al., 2014). Geiger et al. (2016) found that mindfulness-based interventions improved multiple aspects of emotional wellbeing including anxiety, depression, stress, and pain acceptance $>$ Mindfulness can improve pain management associated with chronic pain and reduce stress-related outcomes and disease in chronic illness populations (Creswell et al., 2019).

\subsection{Purpose of the study}

Disease characteristics are largely unchangeable and can have a negative impact on well-being. Psychological flexibility and mindfulness are skills that can be taught and can improve physical and psychological wellness and decrease symptom severity in individuals with incurable chronic illnesses such as FMS (Wicksell et al., 2012). Therefore, our goal was to examine if psychological flexibility and mindfulness predict physical and psychological wellness beyond the effects of disease characteristics and related symptoms, such as pain, depression, and anxiety.

\section{Methods}

We recruited individuals diagnosed with FSSD (FMS, CFS) and AD (MS, RA) from online forums and support groups. Overall, 609 participants completed the study; however, many respondents reported comorbidities and thus, the resulting data analyses were based on 111 participants with only FMS (282 total), 78 with only CFS (212 total), 97 with only MS (116 total) and 103 with only RA (167 total). We used Qualtrics, an online questionnaire platform, for data collection. Individuals were sent to a short description of the study and a link to the consent form After completing informed consent and demographic questions, the remaining questionnaires were presented in random order.

\subsection{Measures}

The Edmonton Symptom Assessment Scale (ESAS; Chang et al., 2000) was used to assess the severity of self-reported medical symptoms. The ESAS is a 9-item self-report scale in which the current severity of symptoms is rated on a 0 (not experienced) to 10 (most severe) scale. Items include physical symptoms such as pain and tiredness, and psychological symptoms such as depression, and well-being). ESAS: Physical was defined as the mean of the items focused on physical wellness (nausea, shortness of breath, appetite, pain, fatigue). The Satisfaction with Life Scale (SWLS) assesses global satisfaction with life (Diener et al., 1985). It contains five statements and uses a Likert scale ranging from 1 (strongly disagree) to 7 (strongly agree), with high scores indicating high satisfaction with life. Generalized Anxiety Disorder -7 (GAD-7; Spitzer et al., 2006), is a seven-item scale that assesses experienced anxiety symptoms in the last two weeks. Individual item scores range from 0 (not at all) to 3 (nearly every day). The Patient Health Questionnaire Mood Scale (PHQ-9; Martin et al., 2006) was used to assess self-reported depression. Scores can be divided into four categories ranging from major depressive 
disorder (most severe), other depressive disorder depressive-screen positive (DS + ) and depressive-screen negative (DS). Participants rated their subjective depression in the last two weeks from 1 (not at all) to 7 (nearly half of all days) on nine items such as, "feeling down, depressed, or hopeless". The Adolescent and Adult Mindfulness Scale (AAMS; Droutman et al., 2018) measures key components of mindfulness which includes a focus of the present moment (AAMS: AA), being nonreactive (AAMS: NonR), being non-judgmental (AAMS: NonJ), and self-acceptance (AAMS: Acc). It consists of 24 statements such as "I notice when my moods begin to change," with individual scores ranging from 1 (never true) to 5 (always true), with higher scores indicating increased mindfulness. Psychological flexibility was assessed using the Comprehensive Assessment of Acceptance and Commitment Therapy processes (CompACT; Francis et al., 2016). This comprehensive measure of psychological flexibility is theorized using the ACT model (Hayes et al., 2016) and contains 23-items scored on a Likert scale from 1 (strongly disagree) to 7 (strongly agree), where high scores equate with high psychological flexibility. Subscale scores include Openness to Experience (CompACT: OE), Behavioural Awareness (CompACT: BA), and Valued Action (CompACT: VA).

\section{Results}

Descriptive statistics on the variables of interest are presented in Table 1. Overall, compared to the AD group, the FSS group had higher levels of ESAS-Physical and PHQ-9, as well as lower SWLS. These scores indicate important differences between the groups in terms of overall depression and life satisfaction. Individuals who have been diagnosed with a FSSD have moderate levels of depression (Martin et al., 2006) and life satisfaction that is lower than average.

Table 1. Mean and standard deviation of Variables Associated with Physical and Psychological Wellness.

\begin{tabular}{|c|c|c|c|c|c|}
\hline & $\begin{array}{r}F \\
\text { Sym }\end{array}$ & $\begin{array}{l}\text { al Son } \\
\text { sordei }\end{array}$ & We & $\begin{array}{l}\text { Autoimmune } \\
\text { rs (AD) }\end{array}$ & $t(p)$ \\
\hline & $\mathrm{M}$ & $s$ & $\mathrm{M}$ & $s$ & \\
\hline SWLS & 19.14 & 1.37 & 23.21 & 10.41 & $-3.77 * * *$ \\
\hline PHQ-9 & 12.29 & 5.20 & 9.98 & 5.44 & $-3.74 * * *$ \\
\hline GAD-7 & 1.39 & 0.78 & 1.23 & 0.76 & -1.76 \\
\hline ESAS-Physical & 4.51 & 1.67 & 3.70 & 1.91 & $-3.92 * * *$ \\
\hline AAMS: AA & 3.53 & 0.82 & 3.28 & 0.79 & $-2.76 * *$ \\
\hline AAMS: NonR & 2.95 & 1.17 & 3.07 & 1.19 & 0.941 \\
\hline AAMS:NonJ & 3.23 & 0.87 & 3.36 & 0.81 & 1.43 \\
\hline AAMS:Acc & 3.57 & 1.00 & 3.74 & 0.86 & 1.56 \\
\hline CompACT: OE & 3.99 & 1.17 & 4.03 & 0.95 & 0.341 \\
\hline CompACT:BA & 2.89 & 0.98 & 2.87 & 1.00 & -0.135 \\
\hline CompACT:VA & 3.92 & 1.29 & 3.78 & 1.35 & -0.887 \\
\hline
\end{tabular}

There were statistically significant correlations between mindfulness, psychological flexibility, and wellness (see Table 2). Both AAMS (apart from Acting with Awareness) and CompACT subscales were consistently associated with anxiety (GAD-7), depression (PHQ-9), and self-reported medical symptoms (ESAS-Total). The associations between mindfulness and psychological flexibility were more variable, especially in participants who reported a FSSD. To examine if mindfulness and psychological flexibility contributed to physical and psychological wellness, two hierarchical regressions were conducted. In both regressions, Block 1 included demographic variables (biological sex, age, illness category), Block 2 included measures of wellness (PHQ-9, GAD-7, SWLS or ESAS: Physical), and Block 3 included AAMS and CompACT subscales. The first regression predicting ESAS: Physical was statistically significant, $\mathrm{F}(13,272)=12.15, \mathrm{p}<.001, \mathrm{R}^{2}=.38$. Block 1 contributed $7.7 \%$ of the variability; being female and having an FSSD were associated with higher ESAS: Physical. Block 2 contributed $35.6 \%$ of the variability; increased PHQ-9 and GAD-7 and lower SWL was associated with higher ESAS: Physical. Block 3 added an additional $2.3 \%$ of the variability, with PF: BA adding to the model. The regression to predict psychological wellbeing (SWLS) was also statistically significant, $\mathrm{F}(13,272)=$ $11.06, \mathrm{p}<.001, \mathrm{R}^{2}=.36$. Block 1 contributed $4.2 \%$ of the variability in SWLS; individuals with FSSD reported lower SWL. Block 2 contributed $21 \%$ of the variability, with higher ESAS: Physical and PHQ-9 scores predicting lower SWL. Block 3 contributed an additional $10.4 \%$ of the variability, with PF: VA adding significantly to the model. 
Table 2. Correlations between AAMS subscales and wellbeing.

\begin{tabular}{|l|llllllll|}
\hline \multirow{4}{*}{ Mindfulness } & \multicolumn{2}{|c}{ GAD-7 } & \multicolumn{2}{c}{ PHQ-9 } & \multicolumn{3}{c|}{ SWL } & \multicolumn{2}{c|}{ ESAS-Physical ${ }^{1}$} \\
\cline { 2 - 9 } & FSSD & AD & FSSD & AD & FSSD & AD & FSSD & AD \\
\cline { 2 - 9 } AA & & & & & & & & \\
NonR & .024 & -.116 & .088 & -.078 & .056 & .135 & .008 & -.088 \\
NonJ & $-.495^{* * *}$ & $-.441^{* * *}$ & $-.362^{* * *}$ & $-.344^{* *}$ & .119 & -.006 & $-.332^{* * *}$ & -.152 \\
Acc & $-.261^{* *}$ & $-.171^{*}$ & $-.306^{* * *}$ & -.140 & -.033 & -.055 & -.036 & -.078 \\
PF & $-.602^{* * *}$ & $-.570^{* * *}$ & $-.525^{* * *}$ & $-.566^{* * *}$ & .156 & $.239^{* *}$ & $-.263^{* *}$ & $-.378^{* * *}$ \\
OE & & & & & & & & \\
BA & $-.607^{* * *}$ & $-.603^{* * *}$ & $-.432^{* * *}$ & $-.521^{* * *}$ & .160 & $.375^{* * *}$ & $-.205^{*}$ & $-.398^{* * *}$ \\
VA & $-.378^{* * *}$ & $-.548^{* * *}$ & $-.377^{* * *}$ & $-.525^{* * *}$ & .082 & $.316^{* * *}$ & $-.284^{* *}$ & $-.442^{* * *}$ \\
\hline
\end{tabular}

Note. ${ }^{* * *} p<.001,{ }^{* *} p<.01,{ }^{*} p<.05 .{ }^{l}$ High ESAS scores indicate more severe symptoms.

\section{Discussion}

The goal of the current study was to determine how psychological flexibility and mindfulness were associated with greater physical and psychological wellness in individuals diagnosed with a chronic autoimmune disorder. Although autoimmune diseases, such as MS and RA, can be diagnosed using specific medical tests, the diagnosis of a FSSD involves a method of exclusion via symptom analysis, resulting in a longer time to diagnosis. The uncertainty in diagnosis and ambiguous treatment strategies can have detrimental effects on physical and psychological wellness (Asbring \& Narvanen, 2002. In this study, individuals with FSSD reported more physical and psychological symptoms and had lower subjective well-being than those with AD. Individuals with a FSSD had SWL scores that were significantly lower than individuals reporting an $\mathrm{AD}$, and relative to norms, the scores of individuals with an FSSD were $<20$, indicating problems in at least one area of their life (likely their health; Diener, n.d.)

Certain aspects of mindfulness, including being non-reactive and self-accepting appear to be important in understanding the relationship between physical symptoms, psychological distress, and overall subjective well-being across both illness groups. At the correlational level, there were no significant differences between the illness groups in mindfulness, except for the mindfulness subscale measuring being present and focusing on the current moment. Individuals with AD had made more use of this aspect of mindfulness, which is defined by an awareness of surroundings, thoughts, feelings, and events (Droutman, 2018). Overall, higher mindfulness was inversely related to physical and psychological symptoms and was positively associated with measures well-being.

Psychological flexibility has been shown to mediate the relationship between symptom severity and well-being. Wicksell et al. (2012) found that in fibromyalgia patients, psychological flexibility training decreased pain and psychological symptoms and improved overall quality of life. Although psychological flexibility scores were similar for the individuals with FSS and AD, there were statistically significant relationships between many aspects of physical health, psychological distress, and subjective well-being. Interestingly, in the current study, different aspects of psychological flexibility predicted physical and psychological wellness. Living in accordance with one's values (valued action component of psychological flexibility) was important in predicting subjective well-being (SWLS), which replicates Proctor and Best (2019) who examined the association between PF and wellness in brain injury patients. Interestingly, present moment awareness (CompACT: BA) predicted better physical wellness. These results suggest that different types of $\mathrm{PF}$ are indicative of different aspects of health and suggest that CompACT training should focus on the complex interaction between overall wellness and psychological flexibility.

\section{References}

Aletaha, D., \& Smolen, J., S., (2018) Diagnosis and management of rheumatoid arthritis: A review. The Journal of the American Medical Association, 320(13), 1360-1372.

Åsbring, P., \& Närvänen, A. L. (2002). Women's experiences of stigma in relation to chronic fatigue syndrome and fibromyalgia. Qualitative health research, 12(2), 148-160.

Chang, V. T., Hwang, S.S., \& Feuerman, M. (2000). Validation of the Edmonton Symptom Assessment Scale, Cancer, 88(9), 2164-2171.

Compston, A., \& Coles, A. (2002). Multiple sclerosis. The Lancet, 359(9313): 1221-1231.

Creswell, J.D., Lindsay, E.K., Villalba, D.K., Chin, B. (2019). Mindfulness training and physical health: mechanisms and outcomes. Psychosomatic Medicine, 81(3), 224-232. 
Diener, E. (n.d.). Understanding the Scores on the Satisfaction with Life Scale. Retrieved from: http://labs.psychology.illinois.edu/ ediener/SWLS.html

Diener, E., Emmons, R. A, Larsen, R. J., \& Griffin, S. (1985) The satisfaction with life scale. Journal of Personality Assessment, 49(1), 71-75.

Droutman, V., Golub, I., Oganesyan, A., \& Read, S. (2018). Development and initial validation of the Adolescent and Adult Mindfulness Scale (AAMS). Personality and Individual Differences, 123, 34-43.

Francis, A. W., Dawson, D. L., \& Golijani-Moghaddam, N. (2016). The development and validation of the comprehensive assessment of acceptance and commitment therapy processes (CompACT). Journal of Contextual Behavioral Science, 5(3), 134-145.

Geiger, P. J., Boggero, I. A., Brake, C. A., Caldera, C. A., Combs, H. L., Peters, J. R., \& Baer, R. A. (2016). Mindfulness-based interventions for older adults: A review of the effects on physical and emotional well-being. Mindfulness, 7(2), 296-307.

Hayes, S. C., Luoma, J. B., Bond, F. W., Masuda, A., \& Lillis, J. (2016). Acceptance and commitment therapy: Model, processes and outcomes. Behavioral Research and Therapy, 44(1), 1- 25

Henningsen, P., Zipfel, S., \& Herzog, W. (2007). Management of functional somatic syndromes. The Lancet, 369 (9565), 946-955.

Holmes, G. P., Kaplan, J. E., Gantz, N. M., Komaroff, A. L., Schonberger, L. B., Straus, S. E., Jones, J. F., Dubois, R. E., Cunningham-Rundles, C., Pahwa, S., Tosato, L., Zegans, L. S., Purtilo, D. T., Brown, N., Schooley, R. T., \& Brus, I. (1988). Chronic fatigue syndrome: A working case definition. Annals of Internal Medicine, 108(3), 387-389.

Jellinger, K. A. (1999). The clinical neuropsychiatry of multiple sclerosis. European Journal of Neurology, 6(6), 728-728.

Kong, F., Wang, X., Zhao, J. (2014). Dispositional mindfulness and life satisfaction: the role of self-evaluations. Personality and Individual Differences, 56, 165-169.

Martin, A., Reif, W., Klaiberg, A., \& Braehler, E. (2006). Validity of the Brief Patient Health Questionnaire Mood Scale (PHQ-9) in the general population. General Hospital Psychiatry, 28(1), 71-77.

McCabe, M.P., \& McKern, S. (2002). Quality of Life and multiple sclerosis: Comparison between people with multiple sclerosis and people for the general population. Journal of Clinical Psychology in Medical Settings, 9(4), 287-295.

Mindfulness based interventions for older adults: A review on the effects on physical and emotional well-being. Mindfulness, 7(2), 296-397.

Mohr, D., \& Cox, D. (2001). Multiple sclerosis: Empirical literature for the clinical health psychologist. Journal of Clinical Psychologist, 57(4), 479-499.

Proctor, C \& Best, L. (2019, March 7-9). The utility of Acceptance and Commitment Therapy (ACT) after brain injury [Poster presentation]. International Convention of Psychological Science (ICPS), Paris, France.

Sokka, T., Kautiainen, H., Möttönen, T., \& Hannonen, P. (1999). Work disability in rheumatoid arthritis 10 years after diagnosis. Journal of Rheumatology, 26(8), 1681-1685.

Spitzer, R. L., Kroenke, K., Williams, J. B. W., \& Löwe, B. (2006). A brief measure for assessing Generalized Anxiety Disorder: The GAD-7. Archives of Internal Medicine, 166(10), 1092-1097.

Turk, D. C., Meichenbaum, D., \& Genest, M. (1983). Pain and behavioral medicine: A cognitive-behavioral perspective. New York: Guilford Press.

Wicksell, R. K., Kemani, M., Jensen, K., Kosek, E., Kadetoff, D., Sorjonen, K., Ingvar, M., \& Olsson, G.L. (2012) Acceptance and commitment therapy for fibromyalgia: A randomized controlled trial. European Journal of Pain, 17(4), 599-611.

Wolfe, F., Clauw, D., Fitzcharles, M. A., Goldenberg, D. L., Katz, R. S., Mease, P., Russell, A. S., Russell, I. J., Winfield, J. B., \& Yunus, M. B. (2010). The American College of Rheumatology preliminary diagnostic criteria for fibromyalgia measurement of symptom severity. Arthritis Care and Research, 62(5), 600-610.

Wolfe, F., Egloff, N., \& Häuser, W. (2016). Widespread pain and low widespread pain index scores among fibromyalgia-positive cases assessed with the 2010/2011 fibromyalgia criteria. The Journal of Rheumatology, 43(9), 1743-1748.

Zeidan, F., Grant, J. A., Brown, C. A., McHaffie, J. G., \& Coghill, R. C. (2012). Mindfulness meditation-related pain relief: Evidence for unique brain mechanisms in the regulation of pain. Neuroscience Letters, 520(2), 165-173. 